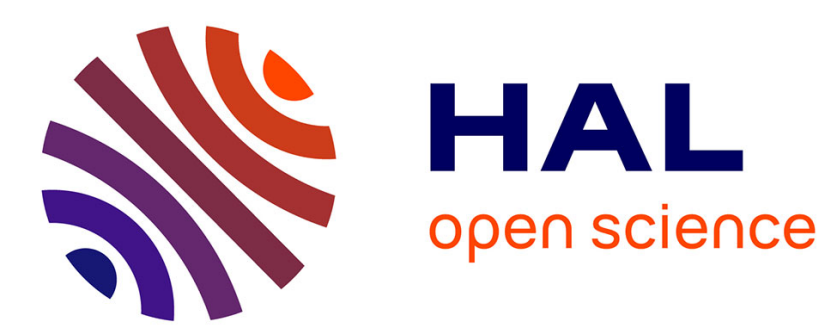

\title{
Compensation of some time dependent deformations in tomography.
}

\author{
Laurent Desbat, Sébastien Roux, Pierre Grangeat
}

\section{To cite this version:}

Laurent Desbat, Sébastien Roux, Pierre Grangeat. Compensation of some time dependent deformations in tomography.. IEEE Transactions on Medical Imaging, 2007, 26 (2), pp.261-9. hal-00292357

\section{HAL Id: hal-00292357 \\ https://hal.science/hal-00292357}

Submitted on 1 Jul 2008

HAL is a multi-disciplinary open access archive for the deposit and dissemination of scientific research documents, whether they are published or not. The documents may come from teaching and research institutions in France or abroad, or from public or private research centers.
L'archive ouverte pluridisciplinaire HAL, est destinée au dépôt et à la diffusion de documents scientifiques de niveau recherche, publiés ou non, émanant des établissements d'enseignement et de recherche français ou étrangers, des laboratoires publics ou privés. 


\title{
Compensation of some time dependent deformations in tomography
}

\author{
Laurent Desbat, Sébastien Roux and Pierre Grangeat, ${ }^{*}{ }^{*}$
}

June 112006

\begin{abstract}
This work concerns $2 D+t$ dynamic tomography. We show that a much larger class of deformations than the affine transforms can be compensated analytically within FBP algorithms in 2D parallel beam and fan beam dynamic tomography. We present numerical experiments on the Shepp and Logan phantom showing that non-affine deformations can be compensated. A generalization to 3D Cone Beam tomography is proposed.
\end{abstract}

\section{Introduction}

This work is a contribution to motion compensation in dynamic tomography [1]. These last years, several studies have been dedicated to this subject, mainly for cardiac imaging but also for patient motion compensation [2, 3]. Indeed, reconstructions of moving organs suffer from artifacts. Gating methods $[4,5,6]$ have been proposed to improve cardiac imaging. CT scanner rotation speed and sampling parameter adjustment to the heart period were also suggested in order to improve the reconstructed images [7].

This paper shares the same approach as Crawford et al [8] and Roux et al [9]. The idea is to introduce a time (denoted by $t$ ) dependent motion or a deformation model $\vec{\Gamma}_{t}$ within the reconstruction. $\vec{\Gamma}_{t}$ is a bijective mapping on the reconstruction space. Let us denote $f_{t}$ the measured attenuation function at time $t$. We suppose that $f_{t}(\vec{x})=f_{\vec{\Gamma}_{t}}(\vec{x}) \stackrel{\text { def }}{=} f\left(\vec{\Gamma}_{t}(\vec{x})\right)$ where $f$ is the attenuation function at a reference time, for example $t=0$. Thus $\vec{\Gamma}_{t}(\vec{x})$ maps $\vec{x}$ at time $t$ to its position at time $t=0$.

In [9] the deformation compensation is incorporated within analytical reconstruction algorithms for time dependent affine deformations $\vec{\Gamma}_{t}$ in 2D parallel

\footnotetext{
${ }^{*}$ L. Desbat is with TIMC-IMAG,UMR UJF-CNRS 5525, Joseph Fourier Grenoble University Faculté de Médecine, 38706 La Tronche, France. Phone +33-4-56-520051. E-mail: Laurent.Desbat@imag.fr.

${ }^{\dagger}$ P. Grangeat is with LETI, CEA-Grenoble, 17 rue des Martyrs 38054 Grenoble, France. E-mail: Pierre.Grangeat@cea.fr.

${ }^{\ddagger}$ Sï£ibastien Roux was with LETI and TIMC IMAG. He is now with Philips Medical Systems Research, Paris, France. E-mail: sebastien.roux@philips.com.

$\S_{\text {This }}$ work is supported by the ACI GRID grant CiGri, the CIMENT project http://ciment.ujf-grenoble.fr/, the Rï£igion Rhï£ine-Alpes grant RAGTIME and the ANR grant ToRIID
} 
and fan beam tomography (a generalization to $3 \mathrm{D}$ is given in [10]). The $2 \mathrm{D}$ fan beam method is based on the recent reconstruction framework proposed by Noo et al [11]. A major idea of [9] is that the line space is invariant by affine transforms. Moreover, parallel lines are transformed into parallel lines and divergent lines are transformed into divergent lines (intersections are preserved).

For the analytic compensation of deformations in dynamic tomography, it seems important to transform the integration lines at time $t$ into lines at time 0 , in order to keep the mathematical properties of line integrals. In the following, we propose to study firstly the time dependent deformations that map in $2 \mathrm{D}$ the set of (parallel) lines orthogonal to the projection direction at time $t$ into a set of lines orthogonal to a (generally) different direction at time 0 and secondly the time dependent deformations that map the divergent beam at time $t$ into a divergent beam at time 0 . In other words, we do not ask our deformations to preserve any lines in the plane (as affine deformations do) but we want only to preserve the acquisition geometry: a parallel geometry projection will be transformed into a parallel geometry projection, a fan beam geometry projection into an other fan beam geometry projection. We show that a subset of these deformations can be analytically compensated. Invertible affine deformations are element of this subset. Deformations from this subset have an infinite number of degrees of freedom whereas invertible affine transforms in the plane have only six degrees of freedom.

In the next section, we introduce our notations in 2D tomography, the parallel and fan beam (divergent) transforms, the time dependent deformations. In section 3, we introduce classes of deformations with infinite number of degrees of freedom (containing affine deformations) that can be analytically compensated within FBP reconstructions (Filtered Back Projection), firstly in parallel and secondly in fan beam tomography. In section 4, we present numerical experiments of the reconstruction of the Shepp and Logan phantom deformed by a non-affine time dependent map. The last section 5 is dedicated to discussions and perspectives.

\section{Deformations preserving the projection geom- etry}

\section{$2.12 \mathrm{D}$ parallel geometry}

We consider the 2D X-ray transform (up to notations, equivalent to the Radon transform in 2D) of a function $f \in \mathbb{L}^{1}\left(\mathbb{R}^{2}\right.$ ) (in the following denoted by parallel geometry)

$$
g_{\mathcal{P}}(\phi, s) \stackrel{\text { def }}{=} \mathcal{P} f(\phi, s) \stackrel{\text { def }}{=} \int_{\mathbb{R}} f(s \vec{\theta}(\phi)+l \vec{\zeta}(\phi)) d l,
$$

where $\phi \in I_{\Phi} \subset \mathbb{R}$ (generally $I_{\Phi}=\left[0, \pi[), s \in I_{S} \subset \mathbb{R}, \vec{\zeta}(\phi)=(-\sin \phi, \cos \phi)^{t} \in\right.$ $S^{1}$, and $\vec{\theta}(\phi)=(\cos \phi, \sin \phi)^{t} \in S^{1}\left(S^{1}\right.$ is the unit circle $)$, see Fig. 1. The function $\mathcal{P}_{\phi} f$ such that $\mathcal{P}_{\phi} f(s) \stackrel{\text { def }}{=} \mathcal{P} f(\phi, s)$, is called the parallel projection at angle $\phi$. The function $f$ has in practice a compact support. We suppose in the following that it is contained in the disk of radius set to 1 as normalization. A projection $\mathcal{P}_{\phi} f$ is not truncated if it is measured for all $s$ such that the line $\vec{\theta}(\phi) \cdot \vec{x}=s$ intersects the support of $f$. If $I_{S}=[-1,1]$ the projections are not 
truncated. The periodicity and symmetry of the Radon transform reads

$$
g_{\mathcal{P}}(\phi, s)=g_{\mathcal{P}}(\phi+2 \pi, s)=g_{\mathcal{P}}(\phi+\pi,-s) .
$$

By Eq. (2), $\mathcal{P}_{\phi} f$ can be reduced, for all $\phi \in \mathbb{R}$, to $\phi \in[0, \pi[$. The function $f$ can be reconstructed from non-truncated projections on $I_{\Phi}=[0, \pi[$.

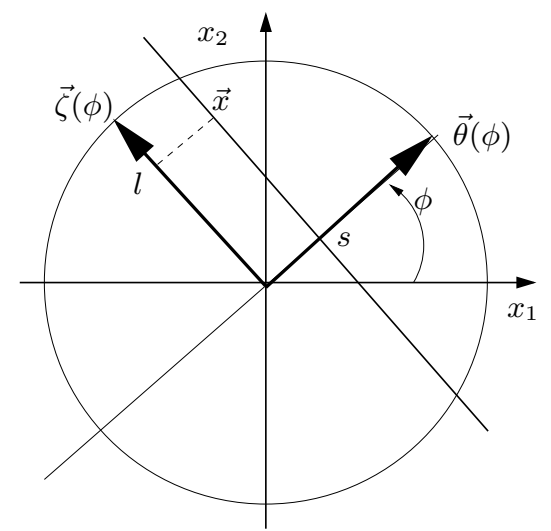

Figure 1: Parallel geometry parameters

\section{$2.22 \mathrm{D}$ fan beam geometry}

We consider the 2D divergent beam, or the fan beam transform, of a function $f \in \mathbb{L}^{1}\left(\mathbb{R}^{2}\right)$ :

$$
g_{\mathcal{D}}(\beta, \alpha) \stackrel{\text { def }}{=} \mathcal{D} f(\beta, \alpha) \stackrel{\text { def }}{=} \int_{0}^{+\infty} f(\vec{a}(\beta)+l \vec{\zeta}(\alpha)) d l,
$$

where $\vec{a}(\beta)$ is the source position, $\beta \in I_{B} \subset \mathbb{R}$ is the real parameter of the source trajectory, $\vec{\zeta}(\alpha) \in S^{1}$, is the unitary vector of the integration half line passing through the source position $\vec{a}(\beta)$ and a detector parametrized by the angle $\alpha \in[-\pi / 2, \pi / 2]$ ( $\alpha$ is the angle between the integration line and the line joining $\vec{a}(\beta)$ and the origin, see Fig. 2). The function $\mathcal{D}_{\beta} f$ such that $\mathcal{D}_{\beta} f(\alpha) \stackrel{\text { def }}{=} \mathcal{D} f(\beta, \alpha)$, is called the fan beam projection at source position $\vec{a}(\beta)$.

In a recent work [11], it has been shown that a point $\vec{x}$ can be reconstructed from non-truncated fan-beam projections acquired from the source trajectory $\vec{a}(\beta), \beta \in I_{B} \subset \mathbb{R}$, provided all line passing through a neighborhood of $\vec{x}$ intersect the source trajectory.

\subsection{Time dependent deformations preserving the $2 \mathrm{D}$ pro- jection geometry}

We study time dependent deformations, more precisely bijective mappings on the plane $\mathbb{R}^{2}$,

$$
\begin{aligned}
& \vec{\Gamma}_{t}: \mathbb{R}^{2} \longrightarrow \mathbb{R}^{2} \\
& \vec{x} \longrightarrow \vec{\Gamma}_{t}(\vec{x})
\end{aligned}
$$




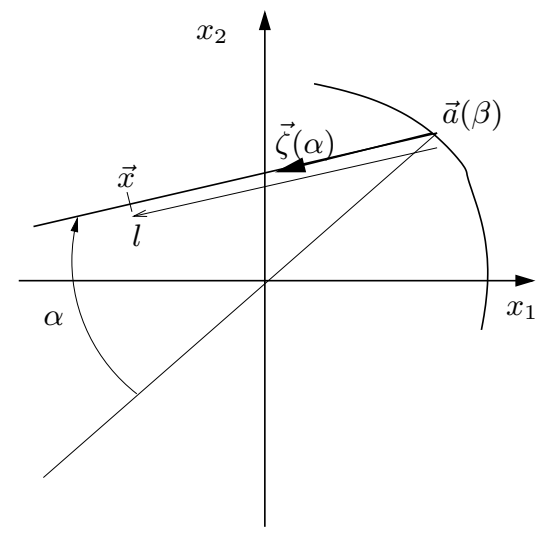

Figure 2: Fan beam (divergent) geometry parameters

The time $t$ will be in practice the parameter characterizing the projection, i.e., the angle $\phi$ for the 2D parallel acquisition (we will then write $\vec{\Gamma}_{\phi}$ ) and $\beta$ for $2 \mathrm{D}$ divergent tomography (we will then write $\vec{\Gamma}_{\beta}$ ) .

\subsubsection{D parallel geometry}

For parallel beams, we consider deformations that transform the set of parallel lines of a projection at angle $\phi$ into a set of parallel lines. Thus there exists an angle $\psi(\phi)$ such that the line $\vec{x} \cdot \vec{\theta}(\phi)=s$ is mapped onto the line

$$
\vec{\Gamma}_{\phi}(\vec{x}) \cdot \vec{\theta}(\phi+\psi(\phi))=\Gamma_{S, \phi}(s) .
$$

This equation means that the line of co-direction $\vec{\theta}(\phi)$ at signed distance $s$ from the origin is mapped by $\vec{\Gamma}_{\phi}$ onto the line of co-direction $\vec{\theta}(\phi+\psi(\phi))$ at signed distance $\Gamma_{S, \phi}(s)$ from the origin, see Fig. 3 . The real function $\Gamma_{S, \phi}$ must be bijective from $I_{S}$ to $\Gamma_{S, \phi}\left(I_{S}\right)$ for $\vec{\Gamma}_{\phi}$ to be bijective. Moreover, we will see in the following that we will need to interpolate $\Gamma_{S, \phi}$, thus we want $\Gamma_{S, \phi}$ to be smooth (it must be continuous but higher regularity improves the interpolation errors). In the following, we will suppose that $\Gamma_{S, \phi}$ is a strictly increasing smooth function.

The deformation $\vec{\Gamma}_{\phi}$ can be written

$$
\vec{\Gamma}_{\phi}(\vec{x})=R_{\psi(\phi)}\left(\Gamma_{S, \phi}(\vec{x} \cdot \vec{\theta}(\phi)) \vec{\theta}(\phi)+\Gamma_{L, \phi}(\vec{x}) \vec{\zeta}(\phi)\right)
$$

where $R_{\psi}$ is the rotation of angle $\psi, \psi$ is a real function from $I_{\Phi}$ to $\left.\psi\left(I_{\Phi}\right)\right) \subset \mathbb{R}$, $\Gamma_{L, \phi}(\vec{x})$ is a real function from $\mathbb{R}^{2}$ to $\mathbb{R}$. With a small abuse of notation, i.e., replacing $\vec{x}$ by its coordinates $(s, l)$ in the basis $(\vec{\theta}, \vec{\zeta}), \Gamma_{L, \phi}(s, l)$ must be a bijection in the second variable $l$ at each fixed $s$ so that $\Gamma_{S, \phi}(s) \vec{\theta}(\phi)+\Gamma_{L, \phi}(s, l) \vec{\zeta}$ is a bijective deformation of the line $s \vec{\theta}(\phi)+l \vec{\zeta}(\phi), \forall l \in \mathbb{R}$. We use the index $L$ in $\Gamma_{L, \phi}$ to underline that the deformation $\Gamma_{L, \phi}$ is according to the parameter $l$ in the $\vec{\zeta}$ direction (just as $\Gamma_{S, \phi}$ is a deformation according to the parameter $s$ 
in the $\vec{\theta}$ direction). However, $\Gamma_{L, \phi}$ may depend on $s$, i.e., vary according to the lines $\vec{x} \cdot \vec{\theta}(\phi)=s$.

Thus, $\vec{\Gamma}_{\phi}$ can be decomposed into two steps

- First, the deformation $\vec{\Delta}_{\phi}$ that preserves the projection direction $\vec{\theta}(\phi)$

$$
\vec{\Delta}_{\phi}(\vec{x}) \stackrel{\text { def }}{=} \Gamma_{S, \phi}(\vec{x} \cdot \vec{\theta}(\phi)) \vec{\theta}(\phi)+\Gamma_{L, \phi}(\vec{x}) \vec{\zeta}
$$

Indeed, let $\vec{y}$ such that $\vec{\Gamma}_{\phi}(\vec{x})=R_{\psi(\phi)} \vec{y}$ then Eq. (5) is equivalent to

$$
\vec{y} \cdot \vec{\theta}(\phi)=\Gamma_{S, \phi}(s)
$$

thus $\vec{y}=\Gamma_{S, \phi}(s) \vec{\theta}(\phi)+\lambda_{\phi}(\vec{y}) \vec{\zeta}(\phi)$ for some real $\lambda_{\phi}(\vec{y})$. As $\vec{y}=R_{-\psi(\phi)} \vec{\Gamma}_{\phi}(\vec{x})$, in Eq. (6) $\Gamma_{L, \phi}(\vec{x})$ is just $\lambda_{\phi}\left(R_{-\psi(\phi)} \vec{\Gamma}_{\phi}(\vec{x})\right)$.

- Then, the rotation $R_{\psi(\phi)}$ of angle $\psi(\phi)$.

\subsubsection{Fan beam geometry}

For fan beam geometry, we will consider deformations $\vec{\Gamma}_{\beta}$ that transform a fan into a fan. Just like for the parallel geometry, we decompose such deformations into two parts:

- First, a deformation that preserves the source position $\vec{a}(\beta)$, i.e., a point $\vec{x}$ of polar coordinates $(l, \alpha)$ with respect to $\vec{a}(\beta)(\vec{x}=\vec{a}(\beta)+l \vec{\zeta}(\alpha))$, is transformed into a point $\vec{\Delta}_{\beta}(\vec{x})$ of polar coordinates $\left(\Gamma_{\mathrm{L}, \beta}(l, \alpha), \Gamma_{\mathrm{A}, \beta}(\alpha)\right)$ :

$$
\vec{\Delta}_{\beta}(\vec{x}) \stackrel{\text { def }}{=} \vec{a}(\beta)+\Gamma_{\mathrm{L}, \beta}(l, \alpha) \vec{\zeta}\left(\Gamma_{\mathrm{A}, \beta}(\alpha)\right) .
$$

The real function $\Gamma_{\mathrm{A}, \beta}$ represents the deformation in the angular direction $\alpha$. At fixed angle $\alpha$, the deformation $\Gamma_{\mathrm{L}, \beta}$ represents the deformation in the radial direction $l$. $\Gamma_{\mathrm{L}, \beta}$ may depends on $\alpha$, i.e., it may be different from one ray to an other ray.

- Then, a translation $\vec{T}_{\vec{v}(\beta)}$ by the vector $\vec{v}(\beta)=\vec{\Gamma}_{\beta}(\vec{a}(\beta))-\vec{a}(\beta)$. The source path $\vec{a}(\beta)$ is transformed into a virtual source path $\vec{\Gamma}_{\beta}(\vec{a}(\beta))$.

Thus we can write the deformation

$$
\vec{\Gamma}_{\beta}(\vec{x})=\vec{T}_{\vec{v}(\beta)}\left(\vec{a}(\beta)+\Gamma_{\mathrm{L}, \beta}(l, \alpha) \vec{\zeta}\left(\Gamma_{\mathrm{A}, \beta}(\alpha)\right)\right)
$$

where $\vec{v}(\beta)=\vec{\Gamma}_{\beta}(\vec{a}(\beta))-\vec{a}(\beta)$. With these notations, $\left(\Gamma_{\mathrm{L}, \beta}(l, \alpha), \Gamma_{\mathrm{A}, \beta}(\alpha)\right)$ are the polar coordinates of $\vec{\Gamma}_{\beta}(\vec{x})-\vec{v}(\beta)$ in the referential with origin at $\vec{a}(\beta)$, see Fig. 4. Just as previously, we suppose that $\Gamma_{\mathrm{L}, \beta}$ and $\Gamma_{\mathrm{A}, \beta}$ are continuous (we will need to interpolate $\vec{\Gamma}_{\beta}$ ). Clearly, for $\vec{\Gamma}_{\beta}$ in (10) to be bijective, $\Gamma_{\mathrm{A}, \beta}$ must be a strictly monotonic function of $\alpha, \Gamma_{\mathrm{L}, \beta}(l, \alpha)$ must be a strictly monotonic function of $l$ and $\Gamma_{\mathrm{L}, \beta}(0, \alpha)$ must be equal to 0 for all $\alpha$. 


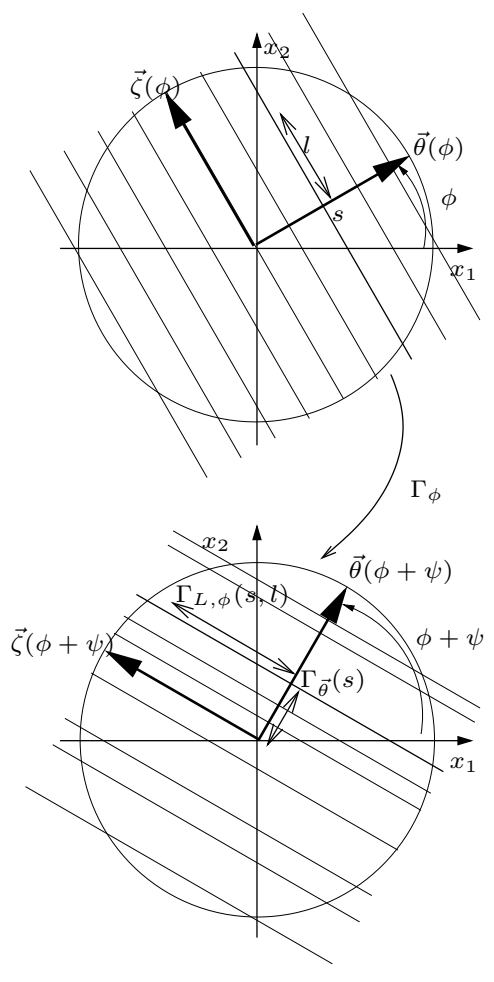

Figure 3: Parallel deformation $\vec{\Gamma}_{\phi}$ : the deformation is a composition of a rotation $R_{\psi}$ and a map from lines $\vec{x} \cdot \vec{\theta}=s$ to lines $\vec{x} \cdot \vec{\theta}=\Gamma_{S, \phi}(s) . \quad l=\vec{x} \cdot \vec{\zeta}(\phi)$ is transformed into $\Gamma_{L, \phi}(\vec{x})$. If, by a small abuse of notations, $\vec{x}$ is replaced in the previous equation by its coordinates $(s, l)$ in the basis $(\vec{\theta}, \vec{\zeta})$, at fixed $s, \Gamma_{L, \phi}(\vec{x})=$ $\Gamma_{L, \phi}(s, l)$ represents the deformation in the direction $\vec{\zeta}$ that transforms $l$ into $\Gamma_{L, \phi}(s, l)$. This function may depend on $s(=\vec{x} \cdot \vec{\theta})$. 


\section{Compensation of time dependent deformations which preserve the projection geometry}

In [9] the function $f$ (and thus $f_{\vec{\Gamma}_{t}}$ ) is analytically reconstructed with FBP algorithms from a parallel or a divergent beam transform $\mathcal{T} f_{\vec{\Gamma}_{t}}$ of $f_{\vec{\Gamma}_{t}}$, more precisely from $\mathcal{P}{f_{\vec{\Gamma}_{\phi}}}_{(}(\phi)(\mathcal{T}=\mathcal{P}$ and $t=\phi)$ or $\mathcal{D} f_{\vec{\Gamma}_{\beta}}(\beta, \alpha)(\mathcal{T}=\mathcal{D}$ and $t=\beta)$, when $\vec{\Gamma}_{t}$ is affine. In the following, we consider deformations $\vec{\Gamma}_{t}$ belonging to subclasses of deformations in the form of (6) or (10): $\vec{\Gamma}_{t}=\vec{A}_{t} \circ \vec{\Delta}_{t}$, where $\vec{A}_{t}$ is affine. Moreover, we restrict the deformation along $\vec{\zeta}$ in Eq. (6) to be affine in $l=\vec{x} \cdot \vec{\zeta}$, see Eq. (12), or in Eq. (10) to be linear in $l$, see Eq. (18). This property is obviously satisfied by the affine deformations, see section 3.3. We will see in sections 3.1 and 3.2 that in this case, $\vec{\Delta}_{t}$ can be analytically compensated at fixed $t$ within each projection $\mathcal{T}_{t} f_{\vec{\Gamma}_{t}}$. The starting point is the following: as

$$
\begin{aligned}
f_{\vec{\Gamma}_{t}}(\vec{x})=f_{\vec{A}_{t} \circ \vec{\Delta}_{t}}(\vec{x}) & =f\left(\vec{A}_{t}\left(\vec{\Delta}_{t}(\vec{x})\right)\right) \\
& =f_{\vec{A}_{t}}\left(\vec{\Delta}_{t}(\vec{x})\right) \\
& =f_{\vec{A}_{t} \vec{\Delta}_{t}}(\vec{x}),
\end{aligned}
$$

the compensation of $\vec{\Delta}_{t}$ in the projections $\mathcal{T}_{t} f_{\vec{\Gamma}_{t}}$ at fixed $t$ yields the projections $\mathcal{T}_{t} f_{\vec{A}_{t}}$. Then, the affine deformation $\vec{A}_{t}$ can be compensated from [9]. Thus, the class of deformation $\vec{\Gamma}_{t}=\vec{A}_{t} \circ \vec{\Delta}_{t}$ can be compensated within analytic reconstruction algorithms like FBP.

\subsection{D parallel geometry}

Let us restrict the deformation $\vec{\Delta}_{\phi}$ to be affine in $\vec{x} \cdot \vec{\zeta}(\phi)$ along the direction $\vec{\zeta}(\phi)$ within each line $\vec{x} \cdot \vec{\theta}(\phi)=s$, i.e., let us replace in Eq. (7) $\Gamma_{L, \phi}(\vec{x})$ by $b_{\phi}(\vec{x} \cdot \vec{\theta}(\phi))+c_{\phi}(\vec{x} \cdot \vec{\theta}(\phi))(\vec{x} \cdot \vec{\zeta}(\phi))$ or equivalently, with $s=\vec{x} \cdot \vec{\theta}(\phi)$ and $l=\vec{x} \cdot \vec{\zeta}(\phi)$ (and a small abuse of notation denoting $\Gamma_{L, \phi}(s, l)$ for $\Gamma_{L, \phi}(\vec{x})$ ) $\Gamma_{L, \phi}(s, l)$ is replaced by $b_{\phi}(s)+c_{\phi}(s) l$. Thus let us consider :

$$
\begin{aligned}
\vec{\Delta}_{\phi}(\vec{x})= & \Gamma_{S, \phi}(\vec{x} \cdot \vec{\theta}(\phi)) \vec{\theta}(\phi)+ \\
& \left(b_{\phi}(\vec{x} \cdot \vec{\theta}(\phi))+c_{\phi}(\vec{x} \cdot \vec{\theta}(\phi))(\vec{x} \cdot \vec{\zeta}(\phi))\right) \vec{\zeta}(\phi)
\end{aligned}
$$

where $c_{\phi}$ is a real strictly positive function then

$$
\mathcal{P}_{\phi} f_{\vec{\Delta}_{\phi}}(s)=\frac{1}{c_{\phi}(s)} \mathcal{P}_{\phi} f\left(\Gamma_{S, \phi}(s)\right)
$$

Indeed

$$
\begin{aligned}
& \mathcal{P} f_{\vec{\Delta}_{\phi}}(\phi, s) \\
= & \int_{\mathbb{R}} f\left(\Gamma_{S, \phi}(s) \vec{\theta}(\phi)+\left(b_{\phi}(s)+c_{\phi}(s) l\right) \vec{\zeta}(\phi)\right) d l \\
= & \frac{1}{c_{\phi}(s)} \mathcal{P} f\left(\phi, \Gamma_{S, \phi}(s)\right) .
\end{aligned}
$$


Thus if we restrict in $(6) \Gamma_{L, \phi}(\vec{x})$ to functions of the form

$$
\Gamma_{L, \phi}(\vec{x})=b_{\phi}(\vec{x} \cdot \vec{\theta}(\phi))+c_{\phi}(\vec{x} \cdot \vec{\theta}(\phi)) \vec{x} \cdot \vec{\zeta}(\phi),
$$

as $\vec{\Gamma}_{\phi}=R_{\psi(\phi)} \circ \vec{\Delta}_{\phi}$, we see from (11) and (13) that

$$
\mathcal{P} f_{\vec{\Gamma}_{\phi}}(\phi, s)=\mathcal{P} f_{R_{\psi(\phi)} \circ \vec{\Delta}_{\phi}}(\phi, s)=\frac{1}{c_{\phi}(s)} \mathcal{P} f_{R_{\psi(\phi)}}\left(\phi, \Gamma_{S, \phi}(s)\right) .
$$

Thus $\mathcal{P}_{\phi} f_{R_{\psi(\phi)}}$ can be computed from $\mathcal{P}_{\phi} f_{\vec{\Gamma}_{\phi}}$ with:

$$
\mathcal{P} f_{R_{\psi(\phi)}}(\phi, s)=c_{\phi}\left(\Gamma_{S, \phi}^{-1}(s)\right) \mathcal{P} f_{\vec{\Gamma}_{\phi}}\left(\phi, \Gamma_{S, \phi}^{-1}(s)\right) .
$$

Then, $f$ can be reconstructed from $\mathcal{P}_{\phi} f_{R_{\psi(\phi)}}, \forall \phi \in I_{\phi}$, with the FBP algorithm given in [9], provided the projections $P f_{\vec{\Gamma}_{\phi}}$ are non-truncated and the image of $I_{\Phi}$ by $I+\psi$ (modulo $\pi$ ) is equal to $[O, \pi$ [. Indeed, $f$ can be reconstructed if the non-truncated projections $\mathcal{P}_{\eta} f$ are available for all angle $\eta \in[0, \pi[$. As $\mathcal{P}_{\phi} f_{R_{\psi(\phi)}}=\mathcal{P}_{\phi+\psi(\phi)} f$, from Eq. (17) $\mathcal{P}_{\phi+\psi(\phi)} f\left(\Gamma_{S, \phi}(s)\right) \propto \mathcal{P}_{\phi} f_{\vec{\Gamma}_{\phi}}(s)$, all necessary projection of $f$ are available iff $\forall \eta \in\left[0, \pi\left[, \exists \phi \in I_{\Phi}\right.\right.$ such that $\eta=\phi+\psi(\phi)$ (this relation being read modulo $\pi$ thanks to the symmetry and periodicity of $\mathcal{P}$, see Eq. (2)). Redundancy can occur and must be treated if $I+\psi$ is not injective on $I_{\Phi}$. The corresponding projection will not be truncated if $\forall u \in \mathbb{R}$ such that the line $\vec{x} \cdot \theta(\phi+\psi(\phi))=u$ intersects the convex hull of $f$, there exists $s \in I_{S}$ such that $\Gamma_{S, \phi}(s)=u$. In practice, if the deformed object stays withing the unit disk, no truncation occurs.

\subsection{D fan beam geometry}

We proceed like for parallel geometry restricting the deformation $\vec{\Delta}_{\beta}$ to be linear in $l$ on half lines $\vec{a}(\beta)+l \vec{\zeta}(\alpha), l \in \mathbb{R}^{+}$, i.e., the function $\Gamma_{\mathrm{L}, \beta}$ is supposed to be linear in $l$ at fixed $\alpha$, more precisely $\Gamma_{\mathrm{L}, \beta}(l, \alpha)=c_{\beta}(\alpha) l$ with $c_{\beta}(\alpha)>0$ (so that the transformation is bijective on the semi-line $\vec{a}(\beta)+l \vec{\zeta}(\alpha), l \in \mathbb{R}^{+}$). Thus let

$$
\vec{\Delta}_{\beta}(\vec{x})=\vec{a}(\beta)+c_{\beta}(\alpha) l \vec{\zeta}\left(\Gamma_{\mathrm{A}, \beta}(\alpha)\right),
$$

where $c_{\beta}$ is a strictly positive function then

$$
\mathcal{D}_{\beta} f_{\vec{\Delta}_{\beta}}(\alpha)=\frac{1}{c_{\beta}(\alpha)} \mathcal{D}_{\beta} f\left(\Gamma_{\mathrm{A}, \beta}(\alpha)\right)
$$

Indeed

$$
\begin{aligned}
& \mathcal{D}_{\beta} f_{\vec{\Delta}_{\beta}}(\alpha) \\
= & \int_{0}^{+\infty} f\left(\vec{a}(\beta)+c_{\beta}(\alpha) l \vec{\zeta}\left(\Gamma_{\mathrm{A}, \beta}(\alpha)\right)\right) d l \\
= & \frac{1}{c_{\beta}(\alpha)} \mathcal{D}_{\beta} f\left(\Gamma_{\mathrm{A}, \beta}(\alpha)\right)
\end{aligned}
$$


Thus if we restrict in (10) $\Gamma_{\mathrm{L}, \beta}(l, \alpha)$ to functions of the form $c_{\beta}(\alpha) l$ (linear functions in l), as $\vec{\Gamma}_{\beta}=\vec{T}_{\vec{v}(\beta)} \circ \vec{\Delta}_{\beta}$, from (11) and (19), we have

$$
\begin{aligned}
\mathcal{D}_{\beta} f_{\vec{\Gamma}_{\beta}}(\alpha) & =\mathcal{D}_{\beta} f_{\vec{T}_{\vec{v}(\beta)} \circ \vec{\Delta}_{\beta}}(\alpha) \\
& =\frac{1}{c_{\beta}(\alpha)} \mathcal{D}_{\beta} f_{\vec{T}_{\vec{v}(\beta)}}\left(\beta, \Gamma_{\mathrm{A}, \beta}(\alpha)\right) .
\end{aligned}
$$

Thus $\mathcal{D}_{\beta} f_{\vec{T}_{\vec{v}(\beta)}}$ can be computed from $\mathcal{D}_{\beta} f_{\vec{\Gamma}_{\beta}}(\alpha)$ :

$$
\mathcal{D}_{\beta} f_{\vec{T}_{\vec{v}(\beta)}}(\alpha)=c_{\beta}\left(\Gamma_{\mathrm{A}, \beta}^{-1}(\alpha)\right) \mathcal{D}_{\beta} f_{\vec{\Gamma}_{\beta}}\left(\Gamma_{\mathrm{A}, \beta}^{-1}(\alpha)\right) .
$$

Then, $f$ can be reconstructed from $\mathcal{D} f_{\vec{T}_{\vec{v}(\beta)}}$ with the FBP algorithm given in [9].

As $f_{\vec{T}_{\vec{v}(\beta)}}(\vec{x})=f(\vec{v}(\beta)+\vec{x})$ and $\vec{\Gamma}_{\beta}(\vec{a}(\beta))=\vec{a}(\beta)+\vec{a}(\beta)$, we have from Eq. (21),

$$
\mathcal{D}_{\beta} f_{\vec{\Gamma}_{\beta}}(\alpha)=\frac{1}{c_{\beta}(\alpha)} \int_{0}^{+\infty} f\left(\vec{\Gamma}_{\beta}(\vec{a}(\beta))+l \vec{\zeta}\left(\Gamma_{\mathrm{A}, \beta}(\alpha)\right)\right) d l .
$$

Thus the virtual source path $\vec{\Gamma}_{\beta}(\vec{a}(\beta))$ need to be outside of the convex hull of the support of $f$. From $[9,11]$, a point $\vec{x}$ can be accurately reconstructed from fan-beam projection acquired during a time dependent deformation of type $\vec{\Gamma}_{\beta}$ provided all lines passing through a neighborhood of $\vec{x}$ intersect the virtual trajectory $\vec{\Gamma}_{\beta}(\vec{a}(\beta)), \beta \in I_{B}$.

The previous reconstruction condition is true provided the acquired projections are not truncated. In practice, all acquired projections will be complete as soon as the support of $f_{\vec{\Gamma}_{\beta}}$ (or equivalently the image by $\vec{\Gamma}_{\beta}^{-1}$ of the support of $f$ ) stays in the field of view of the acquisition system. This means that the deformed object stays in the field of view of the scanner during the acquisition.

\subsection{Time dependent $2 \mathrm{D}$ affine deformations}

In this section, we show that time dependent $2 \mathrm{D}$ affine deformations, $\Gamma_{t}(\vec{x})=$ $M_{t} \vec{x}+\vec{b}_{t}$ such that $\operatorname{det} M_{t} \neq 0$, can be written in the form of equation (6) or respectively equation (10) within the subclass defined by equation (12) or respectively equation (18). In this sense, this paper generalizes [9].

\subsubsection{Parallel geometry}

Let $\vec{\Gamma}_{\phi}(\vec{x})=M_{\phi} \vec{x}+\vec{b}_{\phi}$. The coordinate vector of $\vec{x}$ in the basis $(\vec{\theta}, \vec{\zeta})$ is just $R_{\phi}^{t} \vec{x}$, thus $R_{\phi}^{t}\left(M_{\phi} \vec{x}+\vec{b}_{\phi}\right)=R_{\phi}^{t} M_{\phi} R_{\phi} R_{\phi}^{t} \vec{x}+R_{\phi}^{t} \vec{b}_{\phi}$ is the coordinate vector of $\vec{\Gamma}_{\phi}(\vec{x})$ in the orthogonal basis $(\vec{\theta}, \vec{\zeta})$. A $2 \times 2$ matrix can be transformed into a lower triangular matrix, denoted here $L_{\phi}$, with the multiplication by a rotation matrix, denoted here $R_{\psi(\phi)-\phi}$. Applying this to $R_{\phi}^{t} M_{\phi} R_{\phi}$ yields

$$
\begin{aligned}
\vec{\Gamma}_{\phi}(\vec{x}) & =R_{\phi}\left(R_{\psi(\phi)-\phi} L_{\phi} R_{\phi}^{t} \vec{x}+R_{\phi}^{t} \vec{b}_{\phi}\right) \\
& =R_{\psi(\phi)}\left(L_{\phi} R_{\phi}^{t} \vec{x}+R_{\psi(\phi)}^{t} \vec{b}_{\phi}\right)
\end{aligned}
$$




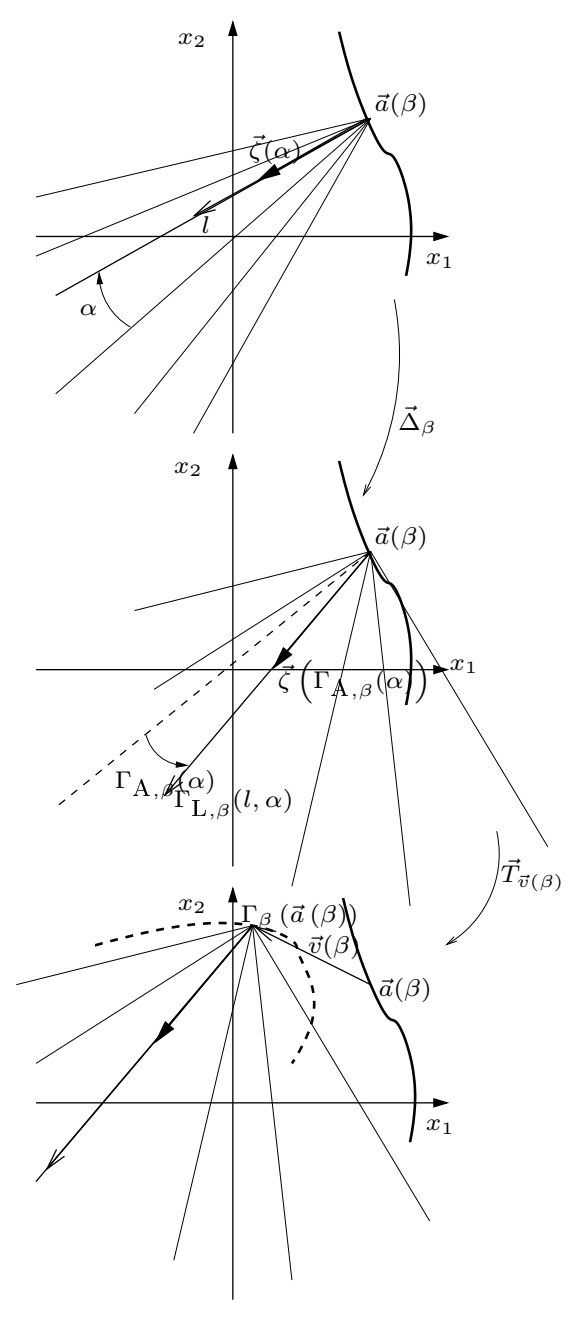

Figure 4: Divergent deformation $\vec{\Gamma}_{\beta}$. First the half lines $\vec{a}(\beta)+l \vec{\zeta}(\alpha), l \in \mathbb{R}^{+}$, are mapped by $\vec{\Delta}_{\beta}$ into the half lines $\vec{a}(\beta)+\Gamma_{\mathrm{L}, \beta}(l, \alpha) \vec{\zeta}\left(\Gamma_{\mathrm{A}, \beta}(\alpha)\right), l \in \mathbb{R}^{+}$, then the whole space is translated by $\vec{v}(\beta)=\vec{\Gamma}_{\beta}(\vec{a}(\beta))-\vec{a}(\beta)$. Note that this translation map the source trajectory $\vec{a}(\beta), \beta \in I_{B}$ into a virtual trajectory $\vec{\Gamma}_{\beta}(\vec{a}(\beta)), \beta \in I_{B}$ at time $t=0$ (represented in the figure as a dashed curve). 
and thus $\vec{\Gamma}_{\phi}$ can be written in the form of the subclass (12) with

$$
\begin{gathered}
\Gamma_{S, \phi}(\vec{x} \cdot \vec{\theta}(\phi))=\left(L_{\phi}\right)_{1,1}(\vec{x} \cdot \vec{\theta}(\phi))+\left(R_{\psi(\phi)}^{t} \vec{b}_{\phi}\right)_{1} \\
b_{\phi}(\vec{x} \cdot \vec{\theta})=\left(L_{\phi}\right)_{2,1}(\vec{x} \cdot \vec{\theta}(\phi))+\left(R_{\psi(\phi)}^{t} \vec{b}_{\phi}\right)_{2} ; c_{\phi}(\vec{x} \cdot \vec{\theta})=\left(L_{\phi}\right)_{2,2}
\end{gathered}
$$

We remark that $\left(L_{\phi}\right)_{2,2}\left(L_{\phi}\right)_{1,1} \neq 0$, which is required for $\Gamma_{S, \phi}$ to be bijective and $c_{\phi}$ to be different from $O$, is equivalent to $M_{\phi}$ is invertible.

\subsubsection{D fan beam geometry}

Let $\Gamma_{\beta}(\vec{x})=M_{\beta} \vec{x}+\vec{b}_{\beta}$. In fan beam, we work with the polar coordinates $(l, \alpha)$ of $\vec{x}$ in the referential centered at $\vec{a}(\beta), \vec{x}=\vec{a}(\beta)+l \vec{\zeta}(\alpha)$, thus $\vec{\Gamma}_{\beta}(\vec{x})=$ $M_{\beta} \vec{a}(\beta)+\vec{b}_{\beta}+l M_{\beta} \vec{\zeta}$. For $\alpha \in[-\pi / 2, \pi / 2]$, the vector $M_{\beta}(\vec{\zeta}(\alpha))$ describes half an ellipsis, thus we can define the one to one function $\Gamma_{\mathrm{A}, \beta}(\alpha)$ of Eq. (18) by

$$
\vec{\zeta}\left(\Gamma_{\mathrm{A}, \beta}(\alpha)\right)=\frac{M_{\beta} \vec{\zeta}(\alpha)}{\left\|M_{\beta} \vec{\zeta}(\alpha)\right\|}
$$

and

$$
c(\alpha)=\left\|M_{\beta} \vec{\zeta}(\alpha)\right\| \text { thus } \Gamma_{\mathrm{L}, \beta}(l, \alpha)=l\left\|M_{\beta} \vec{\zeta}(\alpha)\right\|
$$

which is a linear function of $l$ and generally a non-linear function in $\alpha$. Thus, an affine transform can always be written in the form of Eq. (10) with $\vec{v}(\beta)=$ $\left(M_{\beta}-I\right) \vec{a}(\beta)+\vec{b}_{\beta}$ and $\Gamma_{\mathrm{L}, \beta}(l, \alpha)$ linear in $l$.

\section{Numerical experiments in 2D fan beam}

We consider a scanner of radius $r=3$, the reconstruction region being a disk of radius $\rho=1$, supposed to contain the support of the dynamic function $f_{\vec{\Gamma}_{\beta}}$. Thus, the deformation $\vec{\Gamma}_{\beta}$ is supposed to be a bijection on the unit disk, such that $\mathcal{D}_{\beta} f_{\vec{\Gamma}_{\beta}}$ is completely acquired $\forall \beta \in[0,2 \pi[$. We suppose that the deformation $\vec{\Gamma}_{\beta}$ is equal to $\vec{\Delta}_{\beta}$ with $\vec{\Delta}_{\beta}$ of the form given by Eq. (18), i.e., $\vec{\Gamma}_{\beta}=\vec{T}_{\vec{v}(\beta)} \circ \vec{\Delta}_{\beta}$, with $\vec{v}(\beta)=0$. Thus, we do not consider in this numerical experiment the time dependent affine deformation $\vec{T}_{\vec{v}(\beta)}$ which can be compensated with the FBP algorithm given in [9] (in the case of a translation, this algorithm is particularly simple : it consists simply in the modification of the source trajectory). We consider a deformation $\vec{\Delta}_{\beta}$ with $c_{\beta}(\alpha)=1, \forall \alpha \in\left[-\alpha_{M}, \alpha_{M}\right]$, (where $\alpha_{M}=$ $\arcsin \left(\frac{\rho}{r}\right)=\arcsin \left(\frac{1}{3}\right)$ is half the fan angle), $\forall \beta \in[0,2 \pi[$ and

$$
\begin{aligned}
\Gamma_{\mathrm{A}, \beta}(\alpha)= & \sqrt{\frac{\beta}{2 \pi}}\left(\left(1-\sqrt{\frac{\beta}{2 \pi}}\right) L(\alpha)+\sqrt{\frac{\beta}{2 \pi}} \frac{\alpha^{3}}{\alpha_{M}^{2}}\right) \\
& +\left(1-\sqrt{\frac{\beta}{2 \pi}}\right) \alpha,
\end{aligned}
$$

where $L(\alpha)$ is a piecewise linear strictly increasing function such that $L$ is linear between the points $\left(-\alpha_{M} ;-\alpha_{M}\right),\left(-\alpha_{M} / 2 ;-1.2 \alpha_{M} / 2\right),\left(\alpha_{M} / 2 ; 1.2 \alpha_{M} / 2\right)$, 
$\left(\alpha_{M} ; \alpha_{M}\right)$. Note that we set $\Gamma_{\mathrm{A}, \beta}\left(-\alpha_{M}\right)=-\alpha_{M}$ and $\Gamma_{\mathrm{A}, \beta}\left(\alpha_{M}\right)=\alpha_{M}$ for any $\beta$, so that for any $\beta, f_{\vec{\Gamma}_{\beta}}$ is always in the field of the projection. Our phantom $f$ is essentially the Shepp and Logan phantom. In order to keep the phantom within the unit disk, we have reduced the ellipsis parameter values (the center coordinates and the axis lengths) by the factor 0.8 , but the density of each ellipsis indicator is the same as in the corresponding ellipsis of the Shepp and Logan phantom. In Fig. 5, we present the phantom $f$ and the dynamic phantom $f_{\vec{\Gamma}_{\beta}}$ at different times $\beta=256 / 768 \times 2 \pi, \beta=512 / 768 \times 2 \pi, \beta=767 / 768 \times 2 \pi$ (note that we choose $\vec{\Gamma}_{0}$ to be the identity, thus $f_{\vec{\Gamma}_{0}}=f$, but this is not necessary : $f$ can be considered as a reference. In practice, one would probably consider the reference time in the middle of the $\beta$ interval necessary for the reconstruction, in order to deal with deformations closer to the identity). We obviously can see that the considered deformations are not affine.

The sinograms have been computed with both analytical formulas (using (21) for the dynamic phantom, or equivalently here $(19)$ as $\vec{v}(\beta)=0)$ or discretized line integrals on the pixel grid (in particular for the computation on the dynamic phantom deformed by $\vec{\Gamma}_{\beta}$ ). The acquisition geometry parameters are $P=768$ projections $\left(\beta_{i}=i \frac{2 \pi}{768}, i=0, \ldots, 767\right)$ on a centered FB detector of 512 samples. The phantom is discretized on a $512 \times 512$ pixel image. In Fig. 6 we present the static sinogram $\mathcal{D} f$ of the phantom $f$ computed analytically, the sinogram $\mathcal{D} f_{\vec{\Gamma}_{\beta}}$ of the dynamic phantom computed with line integrals on the dynamic phantom $f_{\vec{\Gamma}_{\beta}}$ (discretized line integrals on the pixelized deformed phantom), the analytic sinogram of the dynamic phantom derived from the static sinogram using formula (19), the difference between the two previous sinograms and finally the sinogram with the deformations compensated with Eq. (22) and the difference between this last compensated sinogram with the static sinogram. We remark that the major errors are located at the boundary of the projection of the ellipsis of higher density, where the interpolation errors are the highest.

In Fig. 7, we compare a reconstruction from static data with reconstructions from dynamic data. We want to show that the deformation compensation with Eq. (22) yields almost the same quality reconstruction as the one we get from static data and improves much compare to a reconstruction from dynamic data without any compensation. The reconstructions are performed with the FBP algorithm using a Shepp and Logan filter given in [12] from data on $\beta \in[0,2 \pi[$. More precisely we have used the FBP algorithm derived from Eq. (1.27a) and (1.27b), page 113, chapter V of [12], and the Shepp and Logan filter given in Eq. (1.23), page 111, with the cut off frequency of the filter equal to $(1+r) \Omega$ where $r=3$ is the radius of the circle trajectory and $\Omega$ the band limit of the reconstructed function is equal to $P / 2=384$. If no deformation compensation is performed then the reconstruction suffers from huge errors, as can be seen in Fig. 7. The artifacts disappears with the reconstruction including the deformation compensation. It appears to be slightly different at the boundary of ellipsis indicators where the interpolation errors of the deformation compensation appear to be the highest. 

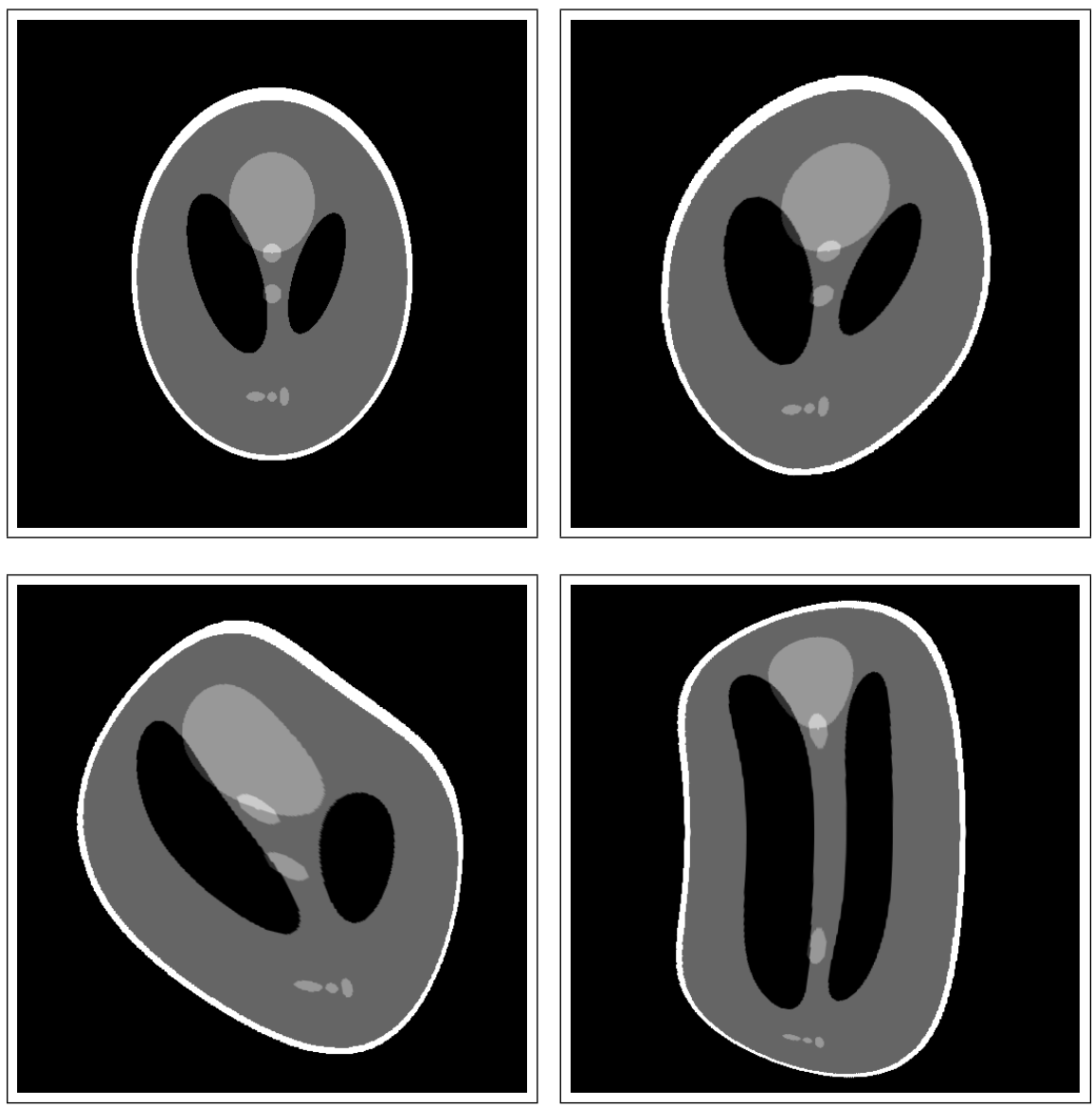

Figure 5: Phantom of the considered dynamic fan-beam deformation. Top left: reference phantom $f$. This is the Shepp and Logan phantom with all geometric dimensions reduced by the factor 0.8 but with the same density values between 0 and 1 . The gray level window is $[0,0.05]$ in all images, 0 being black and 0.05 being white, so that all inner ellipsis can be visible. Top right: deformed phantom $f_{\vec{\Gamma}_{\beta}}$ at time (or angular source position) $\beta=256 / 768 \times 2 \pi$. Bottom left: $f_{\vec{\Gamma}_{\beta}}$ at time $\beta=512 / 768 \times 2 \pi$. Bottom right: $f_{\vec{\Gamma}_{\beta}}$ at time $\beta=767 / 768 \times 2 \pi$. 

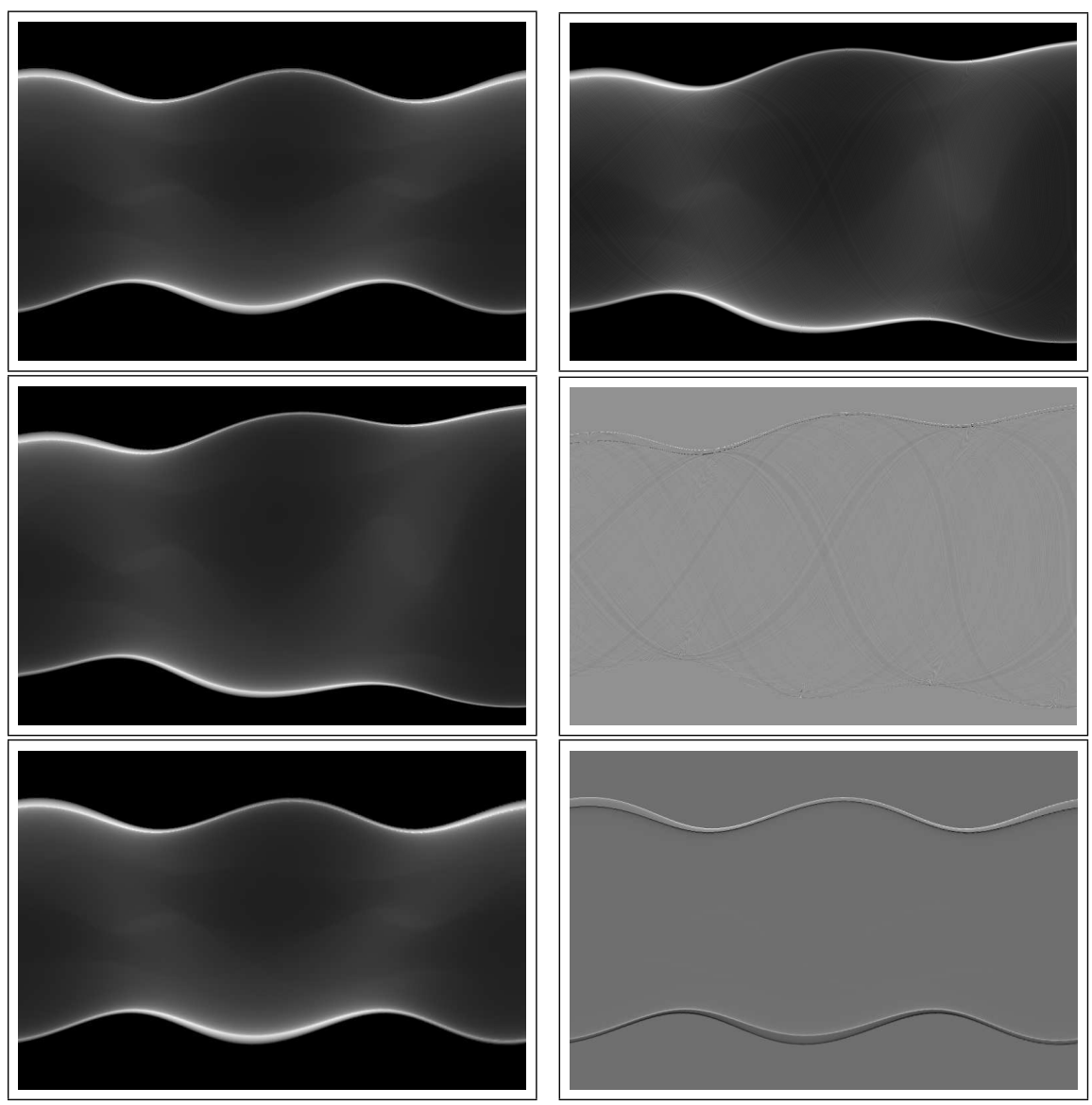

Figure 6: Sinograms in fan beam geometry. First line, left: sinogram of the static phantom. First line, right: sinogram of the dynamic phantom computed from projections of the dynamic phantom (the Shepp and Logan phantom has been pixelized on a $512 \times 512$ image, then this image has been deformed by the $\vec{\Gamma}_{\beta}$ deformation and finally the line integrals have been discretized on the pixel grid of the deformed image, (more precisely the length of the intersection of the integration line with each pixel is multiplied by the pixel density and we sum this result for all pixel intersected by the line). Second line, left: sinogram of the dynamic phantom derived from the sinogram of the static phantom using Eq. (19) with linear interpolation. This three first sinograms are represented with the same gray scale with values from 0 to 0.45 . We see that the two previous dynamic sinograms are close. Second line, right: difference between the two previous sinograms. The minimum is -0.12 and the maximum is 0.09 (and this is the scale too for the gray levels). This large difference is located at the boundary of the large ellipsis. It is due to the fact that a pixellized Shepp and Logan Phantom differs at the boundary of the ellipsis indicators. Moreover the interpolation errors in the computation of Shepp and Logan phantom deformations are also large at the sharp boundary of the ellipsis. Third line, left: corrected dynamic sinogram obtained from Eq. (22) applied to the sinogram of the dynamic phantom derived from the sinogram of the static phantom using Eq. (19) with linear interpolation (second line left) using the same gray levels as for the two previous images. Third 1ime, right: difference between the static sinogram (first line, left) and the corrected dynamic sinogram (Third line, left) [minimum is -0.157 ; maximum is 0.202 , which is also the gray level scale]. Errors are located at the boundary of the projection of the ellipsis. 

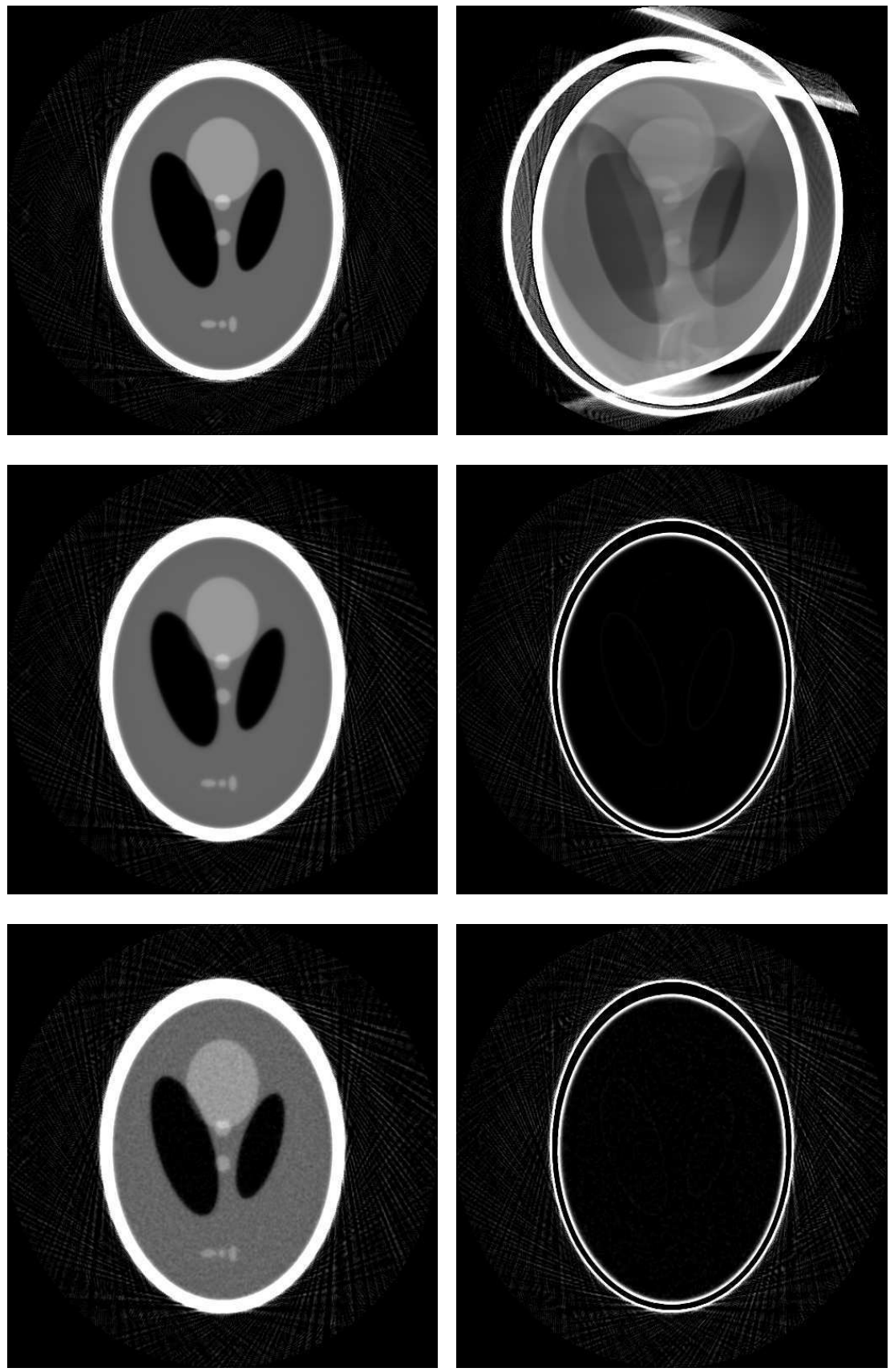

Figure 7: $2 \mathrm{D}$ reconstructions with the FBP algorithm on $[0,2 \pi$ [ given in 'citeNatterer:86 with the Shepp and Logan filter. All the figures are shown with the same gray levels: 0 is black and 0.05 is white. Top left: reconstruction from the static data (Min=-0.017, Max=1.0008). Top right: reconstruction from the dynamic data without deformation compensation (Min $=-0.18, \operatorname{Max}=0,65$, the L2 norm of the difference with left is $1.61 \times 10^{-1}$ ). Second line left: reconstruction from the dynamic data with deformation compensation (Min $=-0.02$, $\mathrm{Max}=0.99$, the L2 norm of the difference with top left is $1.636 \times 10^{-2}$ ). Second line right: difference between the reconstruction from static data and the reconstruction from the compensated dyramic data $(\min =-0,14, \operatorname{Max}=0.11)$. The largest errors are located at the boundary of the ellipsis of high density. Bottom line, similar as the previous line but with noisy data: a Gaussian noise with a standard deviation of $0,2 \%$ of the maximum of the data has been added to the data. Left: $\operatorname{Min}=-0.02, \operatorname{Max}=0.99$. The L2 norm of the difference with top left is $1.64 \times 10^{-2}$. Right: $\operatorname{Min}=-0,15, \operatorname{Max}=0.11$. 


\section{Discussion}

In this work, we have shown that much more general deformations than affine transforms can be analytically compensated in dynamic tomography. We have considered the class of deformations transforming a parallel projection geometry into an other parallel projection geometry, respectively a divergent projection geometry into an other divergent projection geometry. Among these deformations, we have shown that those involving only an affine, respectively a linear, deformation along each line (this affine or linear deformation can vary from line to line), can be efficiently analytically compensated, i.e., within a FBP algorithm. These deformations can be seen as time and line dependent affine, respectively linear, transforms of the object along the integration lines in conjunction with unequally spaced sampling of the parallel, respectively fan beam, projections, combined with a time dependent rotation, respectively a time dependent translation. This class of deformations is much larger than the very small class of bijective affine deformations. Whereas $2 \mathrm{D}$ bijective affine deformations depend only on six parameters, deformations from these new classes have an infinite number of degrees of freedom. Indeed, in parallel geometry, they depend on a rotation angle $\psi$, a bijection $\Gamma_{S, \phi}$ on the parameter $s \in I_{S}$ and affine functions $l \longrightarrow b(s)+c(s) l$, characterized by two scalars $c(s)$ and $b(s)$ (thus two scalar functions $b$ and $c$ of $s \in I_{S}$ ). In fan beam geometry, they depend on a translation vector $\vec{v}$, a bijection $\Gamma_{\mathrm{A}}$ on the angular set $\left[-\alpha_{M}, \alpha_{M}\right]$ and linear functions $l \longrightarrow c(\alpha) l$, characterized by a scalar $c(\alpha)$ (thus a scalar function $c$ of $\alpha \in I_{A}$ ). It allows us for more local deformation possibilities. Part of the organ can stay undeformed (for example in fan beam, lines $\vec{a}(\beta)+l \vec{\zeta}(\alpha)$ for which $\Gamma_{\mathrm{A}, \beta}(\alpha)=\alpha$ and $\left.c_{\beta}(\alpha)=1\right)$. Numerical experiments on the Shepp and Logan phantom have shown in 2D FB geometry that a deformation from this class can be efficiently compensated.

Deformations from the considered classes have been written as a composition of an affine transform (a rotation in parallel geometry or a translation in divergent geometry) and deformations that can be compensated with weighting and rebinning within each projection. Therefore, after this weighting and rebinning step, the admissibility conditions and the FBP algorithms are the same as those given in [9] in 2D.

Just as for affine deformation, the mass of the deformed object can vary when in reality it should generally be conserved, at least in 3D. This question can be addressed in the following way in fan-beam geometry. We suppose first that $\Gamma_{\mathrm{A}, \beta}$ can be extended to a strictly increasing function on $[-\pi ; \pi]$ such that $\Gamma_{\mathrm{A}, \beta}( \pm \pi)= \pm \pi$. The mass $M_{f}$ of $f$ is given by

$$
\begin{aligned}
M_{f} & =\int_{\mathbb{R}^{2}} f(\vec{x}) d \vec{x} \\
& =\int_{-\pi}^{\pi} \int_{0}^{+\infty} f(\vec{a}(\beta)+l \vec{\zeta}(\alpha)) l d l d \alpha
\end{aligned}
$$

The mass $M_{\vec{\Gamma}_{\beta}}$ of $f_{\vec{\Gamma}_{\beta}}$ is given by

$$
\begin{aligned}
M_{f_{\vec{\Gamma}_{\beta}}} & \int_{-\pi}^{\pi} \int_{0}^{+\infty} f\left(\vec{\Gamma}_{\beta}(\vec{a}(\beta))+c_{\beta}(\alpha) l \vec{\zeta}\left(\Gamma_{\mathrm{A}, \beta}(\alpha)\right)\right) l d l d \alpha
\end{aligned}
$$




$$
\begin{aligned}
& =\int_{-\pi}^{\pi} \int_{0}^{+\infty} f\left(\vec{\Gamma}_{\beta}(\vec{a}(\beta))+u \vec{\zeta}\left(\Gamma_{\mathrm{A}, \beta}(\alpha)\right)\right) u d u \frac{1}{c_{\beta}^{2}(\alpha)} d \alpha \\
& =\int_{-\pi}^{\pi} \int_{0}^{+\infty} f\left(\vec{\Gamma}_{\beta}(\vec{a}(\beta))+u \vec{\zeta}(\gamma)\right) u d u \frac{\left(\Gamma_{\mathrm{A}, \beta}^{-1}\right)^{\prime}(\gamma)}{c_{\beta}^{2}\left(\Gamma_{\mathrm{A}, \beta}^{-1}(\gamma)\right)} d \gamma
\end{aligned}
$$

Where we have made the change of the variable $\gamma=\Gamma_{\mathrm{A}, \beta}(\alpha)\left(\Gamma_{\mathrm{A}, \beta}\right.$ is strictly increasing on $[-\pi, \pi]$ ). The mass conservation $M_{f}=M_{{\overrightarrow{\vec{\Gamma}_{\beta}}}}$ will be satisfied for any functions $f$ if the following condition is satisfied:

$$
\frac{\left(\Gamma_{\mathbf{A}, \beta}^{-1}\right)^{\prime}(\gamma)}{c_{\beta}^{2}\left(\Gamma_{\mathbf{A}, \beta}^{-1}(\gamma)\right)}=1 \Longleftrightarrow c_{\beta}^{2}(\alpha) \Gamma_{\mathbf{A}, \beta}^{\prime}(\alpha)=1
$$

Clearly, many other solutions exist so that the mass is conserved. For example, from Eq. (31), we see that multiplying the function $c_{\beta}$ by a constant $k_{\beta}$ will change $M_{\vec{\Gamma}_{\beta}}$ the mass of $f_{\vec{\Gamma}_{\beta}}$ by a factor $\frac{1}{k_{\beta}^{2}}$, thus choosing $\frac{1}{k_{\beta}^{2}}=\frac{M_{f}}{M_{\vec{\Gamma}_{\vec{\Gamma}_{\beta}}}}$ yields the mass conservation. More generally, the conditions $M_{f}=M_{{\overrightarrow{\vec{\Gamma}_{\beta}}}_{\vec{\Gamma}}} \forall \beta \in I_{B}$ can be stated as constraints in the identification of the deformations $\vec{\Gamma}_{\beta}$. However, the mass conservation is not applicable this way in case of truncated projections and movements of organs from inside the field of view to outside the field of view (or reciprocally).

This paper leaves many questions open. More work should be dedicated to the very difficult problem of the deformation estimation, see $[1,3,13]$ for methods and references on this subject. Cardiac or respiration dynamic models could probably help to reach this aim [14]. Having a good estimate of the true deformation, how to compute the best approximation of this deformation in the class of deformations that can be efficiently compensated? How much can we improve the reconstruction this way compared to the improvement from an algebraic approach including the best estimate of the true deformation?

It could be interesting also to search for other deformations that could be analytical compensated. We remark that functions $\Gamma_{L, \phi}(\vec{x})$ of the form $w(\vec{x}$. $\vec{\theta}, \vec{x} \cdot \vec{\zeta}$ ) would yield the rotation invariant Radon transform [15]. Existence and uniqueness conditions for the reconstruction are known but FBP like algorithms are generally not available. We could also consider deformations that transform lines into curves for which inversion formulas exist (such as circles). Then, we could study if (and how) all these deformations could be combined and still be analytically compensated. But this is far beyond the scope of this paper.

Finally, our work could be easily adapted to 3D cone beam geometry. Indeed, let us recall the 3D divergent transform of a real function $f$ of $\mathbb{R}^{3}$,

$$
g_{\mathcal{D}}(\lambda, \vec{\zeta})=\mathcal{D} f(\lambda, \vec{\zeta})=\mathcal{D}_{\lambda} f(\vec{\zeta})=\int_{0}^{+\infty} f(\vec{a}(\lambda)+l \vec{\zeta}) d l
$$

where $\vec{\zeta} \in S^{2}$ is now a unit vector of $\mathbb{R}^{3}, \vec{a}(\lambda) \in \mathbb{R}^{3}$ is a source point $\left(\lambda \in I_{\Lambda} \subset \mathbb{R}\right.$ is the parameter of the source trajectory). Let $(l, \vec{\zeta}) \in \mathbb{R}^{+} \times S^{2}$ be the spherical coordinate of $\vec{x}-\vec{a}(\lambda) \in \mathbb{R}^{3}$, i.e., $\vec{x}=\vec{a}(\lambda)+l \vec{\zeta}$. Let us consider the deformation:

$$
\vec{\Delta}_{\lambda}(\vec{x})=\vec{\Delta}_{\lambda}(\vec{a}(\lambda)+l \vec{\zeta}) \stackrel{\text { def }}{=} \vec{a}(\lambda)+c_{\lambda, \vec{\zeta}} l \vec{\Gamma}_{S^{2}}(\vec{\zeta})
$$


where $c_{\lambda, \vec{\zeta}} \in \mathbb{R}^{+, *}$ and $\vec{\Gamma}_{S^{2}}$ is a bijection on the unit sphere.

$$
\begin{aligned}
\vec{\Gamma}_{S^{2}}: & S^{2} \\
\vec{\zeta} & \longrightarrow \vec{\Gamma}_{S^{2}}(\vec{\zeta})
\end{aligned}
$$

Clearly, the deformation $\vec{\Delta}_{\lambda}$ of Eq. (34) transforms the 3D ray $\vec{a}(\lambda)+\mathbb{R} \vec{\zeta}$ into the $3 \mathrm{D}$ ray $\vec{a}(\lambda)+\mathbb{R}_{S^{2}}(\vec{\zeta})$ and the deformation within the ray is simply linear. Thus, we have

$$
\begin{aligned}
\mathcal{D}_{\lambda} f_{\vec{\Delta}_{\lambda}}(\vec{\zeta}) & =\int_{0}^{+\infty} f\left(\vec{a}(\lambda)+c_{\lambda, \vec{\zeta}} l \vec{\Gamma}_{S^{2}}(\vec{\zeta})\right) d l \\
& =\frac{1}{c_{\lambda, \vec{\zeta}}} \mathcal{D}_{\lambda} f\left(\vec{\Gamma}_{S^{2}}(\vec{\zeta})\right) .
\end{aligned}
$$

Now, from (36) we have the following compensation formula

$$
\mathcal{D}_{\lambda} f(\vec{\zeta})=c_{\lambda, \vec{\Gamma}_{S^{2}}^{-1}(\vec{\zeta})} \mathcal{D}_{\lambda} f_{\vec{\Delta}_{\lambda}}\left(\vec{\Gamma}_{S^{2}}^{-1}(\vec{\zeta})\right)
$$

Just as for the fan beam geometry a global translation of a vector $\vec{v}(\lambda)$ can be composed by the deformation $\vec{\Delta}_{\lambda}$ yielding a 3D deformation preserving the fan-beam geometry

$$
\vec{\Gamma}_{\lambda}(\vec{x})=\vec{T}_{\vec{v}(\lambda)} \circ \vec{\Delta}_{\lambda}(\vec{x})=\vec{a}(\lambda)+\vec{v}(\lambda)+c_{\lambda, \vec{\zeta}} l \vec{\Gamma}_{S^{2}}(\vec{\zeta}) .
$$

From (37) and (11), the deformation $\vec{\Delta}_{\lambda}$ can be compensated leading to

$$
\mathcal{D}_{\lambda} f_{\vec{T}_{\vec{v}(\lambda)}}(\vec{\zeta})=c_{\lambda, \vec{\Gamma}_{S^{2}}^{-1}(\vec{\zeta})} \mathcal{D}_{\lambda} f_{\vec{T}_{\vec{v}(\lambda)} \circ \vec{\Delta}_{\lambda}}\left(\vec{\Gamma}_{S^{2}}^{-1}(\vec{\zeta})\right) .
$$

Now we can simply remark that the time dependent translations by vectors $\vec{v}(\lambda)$ yields the virtual trajectory of the source $\vec{\Gamma}_{\lambda}(\vec{a}(\lambda))=\vec{a}(\lambda)+\vec{v}(\lambda)$. Thus, recent $3 \mathrm{D}$ reconstruction methods $[16,17,18]$ could be adapted: the $3 \mathrm{D}$ reconstruction of $f$ from $\mathcal{D}_{\lambda} f_{\vec{T}_{\vec{v}(\lambda)}}(\vec{\zeta})$ on the vertex path $\vec{a}(\lambda)$ is thus simply the $3 \mathrm{D}$ reconstruction from $\mathcal{D}_{\lambda} f(\vec{\zeta})$ on the vertex path $\vec{\Gamma}_{\lambda}(\vec{a}(\lambda))$.

\section{References}

[1] S. Bonnet, A. Koenig, S. Roux, P. Hugonnard, R. Guillemaud, and P. Grangeat, "Dynamic X-ray computed tomography," Proceedings of the IEEE, vol. 91(10), pp. 1574-87, October 2003.

[2] J. Li, R. J. Jaszczak, H. Wang, and R. Coleman, "A filtered-backprojection algorithm for fan-beam SPECT which corrects for patient motion," Phys. Med. Biol., vol. 40, pp. 283-294, 1995.

[3] P. Grangeat, A. Koenig, T. Rodet, and S. Bonnet, "Theoretical framework for a dynamic cone-beam reconstruction algorithm based on a dynamic particle model," Phys. Med. Biol., vol. 47, no. 15, pp. 2611-2625, August 2002. 


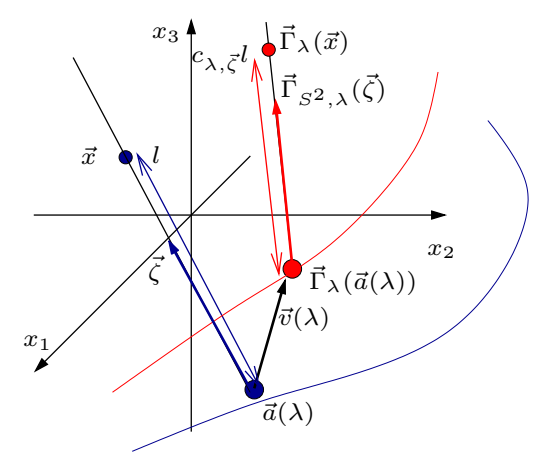

Figure 8: Divergent deformation in 3D preserving the 3D divergent geometry. At time $\lambda$, the point $\vec{x}=\vec{a}(\lambda)+l \vec{\zeta}$ is mapped to $\vec{\Gamma}_{\lambda}(\vec{a}(\lambda))+c_{\lambda, \vec{\zeta}} l \vec{\Gamma}_{S^{2}}(\vec{\zeta})$, i.e., $l$ is changed to $c_{\lambda, \vec{\zeta}}$, the unit direction $\vec{\zeta}$ is mapped to $\vec{\Gamma}_{S^{2}}(\vec{\zeta})$ and then a translation by $\vec{v}(\lambda)=\vec{\Gamma}_{\lambda}(\vec{a}(\lambda))-\vec{a}(\lambda)$ occurs.

[4] M. Kachelriess and W. A. Kalender, "Electrocardiogram-correlated image reconstruction from subsecond spiral computed tomography scans of the heart," Medical Physics, vol. 25(12), pp. 2417-2431, December 1998.

[5] T. Flohr and B. Ohnesorge, "Heart rate adaptative optimization of spatial and temporal resolution for electrocardiogram-gated multislice spiral CT of the heart," Journal of Computer Assisted Tomography, vol. 25(6), pp. 907-923, 2001.

[6] D. R. Gilland, B. A. Mair, J. E. Bowsher, and R. J. Jaszczak, "Simultaneous reconstruction and motion estimation for gated cardiac ECT," IEEE Transactions on Nuclear Sciences, vol. 49, pp. 2344-2349, October 2002.

[7] S. Roux, L. Desbat, A. Koenig, and P. Grangeat, "Efficient acquisition for periodic dynamic CT," IEEE Transactions on Nuclear Sciences, vol. 50(5), pp. 1672-77, October 2003.

[8] C. Crawford, K. King, C. Ritchie, and J. Godwin, "Respiratory compensation in projection imaging using a magnification and displacement model," IEEE Transactions on Medical Imaging, vol. 15, pp. 327 -332, 1996.

[9] S. Roux, L. Desbat, A. Koenig, and P. Grangeat, "Exact reconstruction in 2D dynamic CT: compensation of time-dependent affine deformations," Phys. Med. Biol., vol. 49(11), pp. 2169-82, June 2004.

[10] S. Roux, "Modèles dynamiques en tomographie. application à l'imagerie cardiaque," PhD thesis, Université Joseph Fourier, Grenoble 1, France, 2004.

[11] F. Noo, M. Defrise, R. Clackdoyle, and H. Kudo, "Image reconstruction from fan-beam projections on less than a short-scan," Phys. Med. Biol., vol. 47 , pp. 2525-2546, July 2002.

[12] F. Natterer, The Mathematics of Computerized Tomography. Wiley, 1986. 
[13] W. Lu and T. Mackie, "Tomographic motion detection and correction directly in sinogram space," Phys. Med. Biol., vol. 47, pp. 1267-1284, April 2002.

[14] C. Blondel, R. Vaillant, G. Malandain, and N. Ayache, "3D tomographic reconstruction of coronary arteries using a precomputed 4D motion field," Phys. Med. Biol., vol. 49(11), pp. 2197-2208, June 2004.

[15] E. Quinto, "The Inversibility of Rotation Invariant Radon Transforms," $J$. Math. Anal. Appl., vol. 91, pp. 510-522, 1983.

[16] A. Katsevich, "A general scheme for contructing inversion algorithms for cone beam CT," International journal of Mathematics and Mathematical Sciences, vol. 21, pp. 1305-1321, 2003.

[17] J. Pack and F. Noo, "Cone-beam reconstruction using 1d filtering along the projection of m-lines," Inverse Problems, vol. 21, no. 3, pp. 1105-1120, 2005.

[18] E. Sidky and X. Pan, "A minimum data FBP-type algorithm for image reconstruction in cone-beam CT," in Fully $3 D$ image reconstruction in radiology and medicine proceeding, Salt Lake City, 2005, pp. 291-294. 